patent in $81.3 \%$ of patients one year after implantation. In the light of the tendency for multivessel diseases to increase PTCA will likely be established as an important therapeutic means for ischemic heart disease. CABG, on the other hand, will be employed mainly in elderly heart disease patients and patients with severe multivessel disease or diminished cardiac function, but may meet with less favor than before.

\section{$-303-$ \\ LONG TERM EFFICACY OF PERCUTANEOUS TRANSLUMINAL CORONARY ANGIOPLASTY ASSESSED BY EXERCISE STRESS TEST}

H. Sato, S. Nanto, K. Taniura, Y. Higashino, A. Hirayama, T. Masuyama, K. Komamura, M. Naka, K. Kodama

The Cardiovascular Division, Osaka Police Hospital

Serial bicycle ergometric exercise sessions were scheduled to assess long term exercise performance after successful percutneous transluminal coronary angioplasty (PTCA). Sixtynine patients with effort angina were studied during a mean follow up period of 21 months (range 6 lo 48 months). Exercise stress test were performed before and 7 days; 6 and 12 months; and 2,3 , and 4 years after the intervention. A follow up coronary angiography (CAG) was performed one year after PTCA both in 24 asymptomatic patients and in 8 patients with positive exercise test. In 15 patients regional lactate extraction ratio (LER), calculated with lactate concentration in aorta and great cardiac vein, during atrial pacing stress test (APST) were compared with CAG results and exercise test results. None and 68 of 69 patients were results. None and 68 of 69 patients were
asymptomatic before and 7 days after PTCA, respectively. The average ergometer time increased from 450 sec to 550 sec (before PTCA vs after PTCA: p 0.01), and averaged pressure rate product increase from 194 to 222 units (p 0.01). Two patients redeveloped effort ( $\mathrm{p} 0.01$ ) Two patients redeveloped effort redeveloped angina within one year. The patients without effort angina at one year after PTCA represented coronary stenosis less than $88 \%$ and the changes in the ergometer time did. not correlate with the changes in coronary stenosis. In these patients LER during APST remained in normal range $(22+4 \%)$. Whereas the patients with effort angina at one year after PTCA had severe coronary stenosis greater than $90 \%$, and the ergometer time decreased significantly except one patient. In these patients LER during APST showed 6+58, significantly lower than before APST (p 0.05). Thus we conclude that exercise stress test is a useful method reflecting the long term efficacy of PTCA.

\section{Chairmen}

304-315 K. Kanmatsuse, Tokyo

H. Sato, Hiroshima

\section{$-304-$ \\ BIOCHEMICAL EVALUATION OF INTRACORO- NARY THROMBOLYSIS BY PLASMA CREATINE PHOSPHOKINASE AND SERUM CARDIAC MYOSIN LIGHT CHAIN}

Mitsuaki Isobe, Ryozo Nagai, Yoshio Yazaki Hideaki Nakaoka, Fumimaro Takaku, Tetsu

Yamaguchi*, Hiroki Kurihara*, Masakiyo Nobuyoshi $\S$ Akira Sekif

The Third Department of Internal Medicine,
University of Tokyo, *Mitsui Memorial Hospital, §Kokura Memorial Hospital, fToranomon Hospital

The effects of thrombolytic therapy after acute myocardial infarction (AMI) on infarct size are still controversial. We have reported that serum levels of cardiac myosin light chain (LC) quantitatively reflect infarct size or the impairment of left ventricular function irrespective of the presence of coronary reperfusion. The aim of this study was to evaluate the effects of intracoronary thrombolysis (ICT) using LC and plasma creatine phosphokinase (CPK) activity. Methods Serum LC and plasma CPK were measured serially in 40 patients with AMI who had left anterior descending artery (LAD) lesion and underwent coronary angiography and ICT within 10 hours after the onset. They were divided into 4 groups; A) LAD was already opened at the beginning of ICT $(n=9), B)$ antegrade flow was achieved within 3 hours after the onset $(n=12)$, C) antegrade flow was achieved more than 3 hours after the onset $(n=10)$, and $D)$ antegrade flow could not be achieved $(n=9)$.

Results Peak CPK was appeared earlier in A, B, C than in $D$. Total release of $C P K$ in $C(3099 \pm 1475$, $M \pm S D I U / 1)$ was greater than A $(1431 \pm 947)$, but that in $D(2483 \pm 1481)$ was not greater than that in any other group. Peak LC was appeared about 4 days after the onset in every group. Peak LC in C $(24.9 \pm 6.8 \mathrm{ng} / \mathrm{ml})$ or $D(25.0 \pm 5.2)$ was greater than that in $A(14.8 \pm 6.0)$ or $B(16.1 \pm 7.8)$. This relation was similar when cases were limited to patients with proximal LAD lesion. Mechanical complications such as aneurysm formation and depression of left ventricular ejection fraction tended to be less in A and B. Release of LC was stopped earlier in $A$ and $B$.

Conclusion Limitation of infarct size was achieved by ICT within 3 hours after the onset. Determination of serum LC was useful for the evaluation of the effects of ICT. In contrast, $\mathrm{CPK}$ was washedout after reperfusion.

$-305-$

INFARCT SIZE AND LEFT VENTRICULAR DYSFUNCTION AFTER ACUTE MYOCARDIAL INFARCTION WITH LAD, RCA OR CX OCCLUSION -ASSESSMENT BY TL-201 ECT, $\Sigma$ CK-MB AND GATED CARDIAC POOL SCAN-

Shunichi Tamaki, Shingo Tokunaga, Hirofumi Kambara; Ryuji Nohara\%, Tetsuro Fudo; Tetsuo Hashimoto: Chuichi Kawai. Cardiovascular Div., Takeda Hospital and

The Third Div., Dept. of Internal Med., Kyoto University Hospital?

The relation between an infarct-related coronary artery and infarct size was assessed in 43 cases with first acute myocardial infarction(MI) in whom one coronary artery remained occluded even after intracoronary thrombolysis. Occlusion was $L A D$ in 18, RCA in 15 and $C X$ in 10 patients. Serial CK-MB determinations were used to calculate the

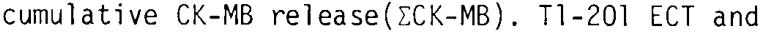
gated cardiac pool scan were performed 4 weeks after the onset to determine scintigraphic infarct size and left ventricular ejection fraction(LVEF). On multiaxial tomograms the size of perfusion defects(PD) was assessed by dividing the LV into 51 myocardial segments scored on a scale of $0-3(0$ : norma 1, 1:smal1 PD, 2:moderate PD, 3: large PD) and summed to form total T1-201 defect score for each patient. Infarct size as assessed by $\Sigma C K-M B$ and T1-201 defect score was significantly larger in $L A D$ group $(406 \pm 203 \mathrm{IU} / \mathrm{L}, 54 \pm 15)$ than in $\mathrm{RCA}(200$ $\pm 74 \mathrm{IU} / \mathrm{L} p<0.001,29 \pm 10 \mathrm{p}<0.001)$ or in $\mathrm{CX}$ group $(203 \pm 110 \mathrm{IU} / \mathrm{L} p<0.02,26 \pm 11 \mathrm{p}<0.001)$. LVEF was more depressed in $L A D$ group $(40 \pm 74 \%)$ than in RCA 